\title{
CODICIA E IRRESPONSABILIDAD, ¿LAS OTRAS CAUSAS DE LAS CRISIS?
}

\section{Pedro Gonzales}

Licenciado en Contabilidad por la Universidad Peruana Unión. Doctor en Administración por la Universidad Nacional Federico Villarreal. Actualmente se desempeña como docente de posgrado de la Universidad de Montemorelos. Asimismo, realizó actividades de docencia en diversos posgrados organizados por dicha universidad. Ha publicado numerosos artículos en revistas nacionales y extranjeras. 


\section{Resumen}

El fracaso de las recetas económicas, poscrisis financieras, implementadas en diversos países del mundo para el rescate de la economía global, el desborde de codicia e irresponsabilidad que se sigue dando en los mercados financieros, el excesivo endeudamiento de los países más desarrollados, la existencia de una economía financiera sobredimensionada frente a la economía real, la inestabilidad persistente del sistema económico y financiero mundial frente a la insolvencia de algunos países, nos ofrecen la oportunidad de explorar nuevos enfoques para afrontar las crisis económicas y financieras. Este estudio aborda, desde una perspectiva no convencional, la existencia de otras causas que alientan este tipo de crisis económica. Se plantean ideas basadas en los principios de una economía solidaria que tiene antecedentes bíblicos y que alude a un mayor compromiso y responsabilidad social por parte de los individuos, corporaciones y países.

Palabras clave: Crisis, economía, depresión, codicia.

\section{Abstract}

The failure of the economic formulas, post crisis financials, applied in several countries in the world aiming for the recovery of the global economy, the overflow of greed and irresponsibility that continues giving to the money market an excessive indebtedness coming from the most developed countries, the existence of an exorbitant financial economy facing to the real economy, constant instability on the economic and financial world system facing to the financial ruin of some countries that offer us opportunities to explore new approaches for facing economic and financial crisis. This text takes from a non conventional way, the existence of other causes that promote this kind of economic crisis, are arisen ideas based in supportive economy principles which has biblical precedents and that refers a greater commitment and social responsibility coming from the individuals, corporations and countries.

Keywords: Crisis, economy, depression, greed. 


\section{Introducción}

En el estudio de las causas de las crisis económicas podemos encontrar una variedad de enfoques que van desde los que se apoyan en los fundamentos de la teoría económica, como aquellos basados en los ciclos económicos, hasta los referidos al estudio del comportamiento de los mercados financieros y su complejo sistema: los productos derivados. El análisis de ello puede conducirnos a diversos planteamientos y propuestas para ofrecer posibles soluciones a esta problemática, propuestas que aun viniendo de los más altos niveles de donde se toman las grandes decisiones económicas, no siempre tienen el efecto esperado, como quedó en evidencia tras la última gran crisis que se inició en el año 2008. El despliegue de enormes cantidades de recursos que se aplicaron en esa ocasión y los que aún se siguen aplicando para el rescate de la economía global, especialmente en los países desarrollados, no ha sido suficiente.

Existen razones para considerar que hoy, a tres años del inicio de la crisis, aún persisten en un alto grado las causas que la impulsaron. Los datos recientes de la economía de Estados Unidos han empeorado, sugiriendo una desaceleración en el crecimiento de un ritmo ya flojo en el primer semestre. Las previsiones para los años siguientes no son favorables. Según Marcet (2010), Estados Unidos se encamina hacia una crisis sin precedentes que golpeará muy fuerte a los hogares y la riqueza ficticia creada en los últimos años se evaporará, trayendo una reducción del nivel de calidad de vida de los americanos.

La preocupación por la existencia de una crisis global de mayores dimensiones subsiste. El sistema financiero internacional y la economía mundial siguen haciendo equilibrio parados en una tenue cuerda floja y rodeados de un vacío abismal. Paul Krugman, Premio Nobel de Economía, la calificó como una crisis económica que será peligrosa, brutal y larga. Él la consideró como la peor crisis económica y financiera desde la Gran Depresión (Krugman, Stiglitz, Gorbachov, Soros, Tofler, Brown, Oppenheimer y otros 2011).

Hace pocos días, en un artículo, en los medios españoles, el secretario general de la OCDE, señaló : “Nos gustaría creer que ya hemos pasado lo peor de la mayor crisis en 70 años, pero los derivados, uno de los principales culpables de la crisis financiera, siguen sumando 10 veces el PBI" (Gurría, 2011). En un artículo, en el diario especializado Financial Times, una de las pocas periodistas que alertó sobre la burbuja financiera que estalló el año 2008, Gillian Tett, advirtió "si no era posible que la crisis de 2008 fuera un 
simple globo de ensayo de la que se viene ahora" (BBC Mundo, 2009). Un informe de la Organización Internacional de Trabajo (OIT) y el Fondo Monetario Internacional (FMI), afirman que el panorama aún se muestra sombrío a pocos meses de iniciar el 2011. Los saldos son desoladores. Desde el 2007 hasta la fecha se agregaron 30 millones de nuevos desempleados alcanzando una cifra acumulada de 210 millones, la más alta de la historia, siendo los países más ricos, con EEUU a la cabeza, los que lideran en el mundo la destrucción del empleo (Fondo Monetario Internacional, 2010). Algunos analistas, como el nobel de Economía Joseph Stiglitz, expresaron su preocupación ante la proyección de la crisis. "El mundo seguro que no saldrá de la crisis ni en el 2010, ni en el 2011. Quizás ni en el 2012. La crisis financiera mundial no se acerca a su fin y no terminará antes del 2013" (British Broadcasting Corporation, BBC, 2009).

La incertidumbre persiste, las gigantescas inyecciones de capital, recurrentes en algunos casos, no han generado los efectos deseados. La deuda soberana de los países desarrollados, la nueva subprime, como algunos analistas la denominan, está sobredimensionada, ha resistido remedios y amenaza con tragarse a grandes economías de la zona euro como España e Italia. Los analistas no vislumbran un panorama alentador, algunos han pronosticado más pérdidas, aun cuando las acciones han caído, a inicios de agosto, en nueve de los últimos diez días. Los rendimientos de las notas del Tesoro a dos años cayeron a un mínimo histórico, dado que los inversores buscaron refugio en la deuda del gobierno de corto plazo. Milton Ezrati, estratega de mercado de Lord Abbett Co., en Jersey City, Nueva Jersey, que gestiona 110.000 millones de dólares en activos, expresó : "La gente se está rindiendo porque no pueden encontrar alivio en ningún frente"(Monn, 2011). En el otro lado del mundo Jyrki Katainen, primer ministro de Finlandia ante la subida de los rendimientos a los máximos históricos de los bonos españoles e italianos, dijo: "esto es algo muy alarmante, toda Europa está en una situación muy peligrosa”, (Reuters, 2011).

Ante este sombrío panorama de la economía global, nos preguntamos:

¿Qué otras causas no convencionales podríamos considerar en el origen de estas crisis?

¿Existen otras posibilidades, fuera de los convencionalismos de la teoría económica, que nos permitan explorar otros tipos de soluciones?

Al iniciar este trabajo planteamos como título: la codicia y la irresponsabilidad como otras de las causas de las crisis económicas; esto nos lleva 
a suponer la existencia de conductas que subyacen en el comportamiento humano y que suponemos han contribuido y seguirán contribuyendo a generar inestabilidad en el endeble sistema económico y financiero mundial.

Abordemos este enfoque citando las declaraciones que dio la ex presidenta de Chile, Michelle Bachelet, en los primeros días que explotó la crisis que aún padecemos. Ella señaló ante el pleno de la $63^{\circ}$ Asamblea General de la ONU, a la que asisten jefes de Estado de Gobierno de 192 países, que la "codicia y la irresponsabilidad de unos pocos han sumido al mundo en la actual crisis financiera" (Seisdedos, 2008). Nos preguntamos, ¿qué tipo de relación existe entre la codicia y la crisis que estamos analizando? ¿Podemos considerar a la codicia como una de las causas del problema? Algunos dirán como el New York Times que el culpable de la crisis tiene un nombre y son los instrumentos financieros derivados, vinculados, en este caso, a las hipotecas subprime o de alto riesgo, que han sido catalogadas por el prominente financista Warren Buffet como "armas de destrucción masiva, con peligros de ser potencialmente letales" (Goodman, 2008: 2). Otros le echan la culpa al elevado déficit de EEUU, el país más endeudado del mundo, quien a estas alturas de la redacción de este documento se debate en la posibilidad de caer en la insolvencia por primera vez en su historia. Según las palabras de Timothy Geithner, el Secretario del Tesoro: “Estados Unidos está al borde de la insolvencia". (Moreno, 2011: 1).

\section{Codicia}

Una de las causas que analizaremos más ampliamente, al abordar este estudio, lo encontramos, como señaló la ex presidenta de Chile, en la conducta humana y es que la codicia y la irresponsabilidad, en este caso, asociada al manejo de las finanzas internacionales, son dos aristas de la misma problemática que se complementan para llevar al ser humano a un afán de riquezas sin límites. Revisemos, en primer lugar, el término codicia. El diccionario (Wordreference, 2011) lo define como: "Deseo, apetito ansioso y excesivo de bienes o riquezas: es un avaro cuya codicia no tiene límites. La Biblia menciona en el libro de Job, hablando de las personas que hacen mal uso de las riquezas: "su ambición nunca quedó satisfecha; nada quedó a salvo de su codicia" (Job: 20: 20), Nueva Traducción Viviente. La palabra codicia también viene de una raíz anglosajona que se refiere al "hambre o apetito". Normalmente tendemos a pensar que la codicia es una compulsión sobrecogedora de obtener riquezas exorbitantes. Se inicia con un pequeño apetito y cuando la persona 
se rinde ante ese poco, se alimenta su codicia y ésta crece un poco más. La codicia nunca se satisface, sino que continúa creciendo y demandando más. De una manera irresponsable el codicioso se concentra insensiblemente en su propia demanda. Los escritos bíblicos abundan en advertencias contra la codicia. Una de ellas está en los diez mandamientos dados por Dios a Moisés y tiene que ver justamente con erradicar la codicia de nuestro corazón y poner fin a su loca carrera autodestructiva (Ex.20:17).

El economista Marcel Claude (citado por Larrabure, 2009: 3) en un artículo del 6 de octubre de 2008 denominado: Crisis del capitalismo, hacia el fin de la era neoliberal nos cuenta de modo muy gráfico y entendible la relación de la codicia con la crisis financiera actual. "Si a la codicia, como valor supremo de la humanidad capitalista le añadimos las características actuales del mercado financiero, podremos comprender mejor las causas de las crisis actual: ¿Para qué ganar 100 si puedes ganar 500? ¿Para qué producir leche si se pueden comprar instrumentos financieros que rinden mucho más y con más celeridad? El primer problema es que los instrumentos financieros no se comen y los tomates sí, las acciones en bolsa no contienen los nutrientes que los niños del mundo necesitan; el segundo problema es que cuando se invierte el dinero en acciones o instrumentos financieros, se deja de invertir en la producción de leche o de trigo. Todo se transforma en un juego, en un simulacro de riqueza. Resultado: menos actividad económica, menos empleo, menos leche y más hambre, más desempleo y más pobreza".

Quedamos asombrados con acontecimientos recientes que se refieren a gigantescas estafas alentadas por la codicia como la cometida por uno de los inversionistas más grandes y famosos de Wall Street, el señor Bernard Madoff, ex presidente de Nasdaq (la segunda Bolsa de Valores de New York) y uno de los "inversores" más destacados en los últimos 50 años. Bernard Madoff fue detenido el 11 de diciembre de 2008 por el FBI, tras confesar que habría causado pérdidas de más de 50.000 millones de dólares a sus clientes. Resulta inconcebible que en pleno siglo XXI, siga funcionando el "Esquema Ponzi", que es un fraude piramidal como el que también fue descubierto en Colombia: la rentabilidad prometida se paga con el dinero ingresado mediante la entrada de nuevos clientes, no por la producción de bienes y servicios.

La codicia no solo es una expresión de la conducta humana sino lo es también de las organizaciones, corporaciones y en un plano mayor de los países. La concentración de la riqueza en el mundo en unos pocos sobrepasando los límites de lo ordinario y la insensibilidad frente al problema de la pobreza. Según la declaración de las Naciones Unidas, emitida como resultado de la 
Cumbre Mundial sobre el Desarrollo Social, en Copenhague, 1995, la pobreza es "una condición caracterizada por la privación severa de las necesidades básicas humanas, tales como alimento, agua potable, facilidades sanitarias, salud, refugio, educación e información. Esta depende no solo del ingreso sino también del acceso a los servicios" (Naciones Unidas [ONU, 2000]).

La brecha de la desigualdad es cada vez más profunda y más amplia entre quienes concentran la riqueza del mundo y los que menos la poseen. Cifras del Banco Mundial para la pobreza en el mundo revelan que cada vez más un mayor número de personas viven en la pobreza. En agosto de 2008, Merrel Tuck (2011) del Banco Mundial presentó una importante revisión de sus estimaciones de la pobreza mundial, la estimación del número de personas que viven con el equivalente de US. 1,25 dólares al día y las cifras son alarmantes. Los datos nos muestran que 1,4 billones de personas, que representan el $20 \%$ de la población mundial, viven o están por debajo de este nivel de pobreza, un 30\% de la población mundial alcanza un promedio de ingreso de US. 2,00 dólares al día y un aproximado de $50 \%$ está en la línea o por debajo de US. 2,50 dólares al día.

Otro estudio publicado por el Instituto Mundial para la Investigación de Desarrollo Económico de la Universidad de las Naciones Unidas (World Institute for Development Economics of the United Nations University [ UNUWIDER]), nos muestra el otro aspecto del problema, el cual es el más completo en cuanto a concentración de la riqueza personal. En ese estudio se reporta que el $2 \%$ de adultos más ricos posee más del $50 \%$ de la riqueza familiar mundial, el $1 \%$ de los adultos más adinerados posee el $40 \%$ de los activos globales y el $1 \%$ de los adultos más adinerados acreditaba un $85 \%$ del total mundial, en contraste, la mitad de la población adulta en el mundo posee escasamente $1 \%$ de la riqueza mundial. La riqueza está fuertemente concentrada en Norteamérica, Europa y países de Asia Pacífico con altos ingresos. La gente en estos países retiene en masa casi un $90 \%$ de la riqueza total del mundo. Aunque Norteamérica solamente tiene el $6 \%$ de la población adulta en el mundo, da cuenta del $34 \%$ de la riqueza familiar. Europa y países de Asia Pacifico con altos ingresos también poseen cantidades desproporcionadas de riqueza (UNU-WIEDER, 2007).

\section{Irresponsabilidad}

Las expresiones de la ex presidenta de Chile, aluden también a la irresponsabilidad como otra causa de las crisis, la codicia lleva implícita la irresponsabilidad y contribuye a profundizar las desigualdades frente a los 
menos favorecidos. El management moderno ha creado el concepto de responsabilidad social, con el fin de generar mecanismos que contribuyan a equilibrar la distribución de la riqueza no solo hacia el desarrollo de las organizaciones mismas sino también hacia su propia gente, su comunidad y el cuidado de su entorno. Bajo este concepto dentro de la administración y del management se engloban un conjunto de prácticas, estrategias y sistemas de gestión empresariales que persiguen un nuevo equilibrio entre las dimensiones económica, social y ambiental. Los antecedentes de la RSE se remontan al siglo XIX en el marco del cooperativismo y el asociacionismo que buscaban conciliar eficacia empresarial con principios sociales de democracia, autoayuda, apoyo a la comunidad y justicia distributiva. Sus máximos exponentes en la actualidad son las empresas de economía social, por definición Empresas Socialmente Responsables. (Tomas, 2003).

\section{Una economía social y solidaria}

El análisis breve que hemos hecho de esta problemática, nos lleva a plantear como dice la Biblia en el libro de Timoteo, que la raíz de todos los males, y en este caso particular de las crisis, es el amor al dinero. La codicia en las dimensiones de este contexto, es una manifestación irresponsable del manejo de la riqueza y el crecimiento económico que contribuye a incrementar la desigualdad. La desigualdad del ingreso o desigualdad económica, comprende todas las disparidades en la distribución de bienes e ingresos económicos. El término se refiere normalmente a la desigualdad entre individuos y grupos al interior de una sociedad, pero también se puede referir a la desigualdad entre países. La desigualdad económica está relacionada con la idea de igualdad de oportunidades y la igualdad de resultados, tanto en términos utilitarios como ético-morales.

En el mundo predomina un modelo económico que pone el acento en el desarrollo básicamente individual, en pos de ganancias principalmente medidas en términos de capital monetario, las teorías y los procesos económicos que nos regulan se han formulado en respuesta a la codicia de las naciones ricas y de los individuos ricos, sin embargo, creemos también en la posibilidad de generar nuevos paradigmas que promuevan el desarrollo de otro tipo de economía, como lo menciona el economista Hiran Sánchez, profesor de la Universidad Autónoma de Santo Domingo, “ ...la economía solidaria debe constituirse en paradigma de los sectores empresariales, sociales e institucionales, debiendo ser la vanguardia del desarrollo" (Economía 
Solidaria, 2010: 8). Una de las características de este tipo de economía como lo menciona Verano es "la supremacía del trabajo sobre el capital, con lo cual se reencuentran el origen y la función de la economía y el desarrollo humano y se rescata el trabajo y su dignidad de la esclavitud ejercida por el capital" (Economía Solidaria, 2010: 7).

Ante el fracaso del sistema económico actual, se necesita una nueva visión heterodoxa de la teoría económica que nos permita incluir esta problemática en el análisis económico, algunos le llaman economía solidaria o economía social; sin embargo, sea cual fuere el nombre, de lo que se trata es lograr un mundo más solidario, como lo expresó D’Escoto al presidir la 63 Asamblea de la ONU : "sustituir el individualismo y egoísmo de la cultura dominante y hacer de la solidaridad humana una norma inquebrantable de conducta". (ONU 2008). En el mundo podemos encontrar ejemplos de ello, el registro bíblico nos refiere uno de tantos casos de economía solidaria, como los que practicaron los macedonios en los inicios del cristianismo, orientados por elevados principios de generosidad y de solidaridad, principios no ortodoxos que las economías del mundo harían bien en alentar y aplicar para enfrentar esta problemática a un nivel personal, corporativo y de país. Veamos este antecedente bíblico, que el apóstol Pablo nos presenta al respecto en su segunda epístola a los Corintios:

\section{Corintios 8: 1-15}

Ahora, hermanos, queremos que se enteren de la gracia que Dios ha dado a las iglesias de Macedonia. ${ }^{2}$ En medio de las pruebas más difíciles, su desbordante alegría y su extrema pobreza abundaron en rica generosidad. ${ }^{3}$ Soy testigo de que dieron espontáneamente tanto como podían, y aún más de lo que podían, ${ }^{4}$ rogándonos con insistencia que les concediéramos el privilegio de tomar parte en esta ayuda para los * santos. ${ }^{5}$ Incluso hicieron más de lo que esperábamos, ya que se entregaron a si mismos, primeramente al Señor y después a nosotros, conforme a la voluntad de Dios. ${ }^{6}$ De modo que rogamos a Tito que llevara a feliz término esta obra de gracia entre ustedes, puesto que ya la había comenzado. ${ }^{7}$ Pero ustedes, así como sobresalen en todo en fe, en palabras, en conocimiento, en dedicación y en su amor hacia nosotros, procuren también sobresalir en esta gracia de dar. ${ }^{8}$ No es que esté dándoles órdenes, sino que quiero probar la sinceridad de su amor en comparación con la dedicación de los demás. ${ }^{9}$ Ya conocen la gracia de nuestro Señor Jesucristo, que aunque era rico, por causa de ustedes se hizo pobre, para que mediante 
su pobreza ustedes llegaran a ser ricos. ${ }^{10}$ Aquí va mi consejo sobre lo que les conviene en este asunto: El año pasado ustedes fueron los primeros no solo en dar sino también en querer hacerlo. ${ }^{11}$ Lleven ahora a feliz término la obra, para que, según sus posibilidades, cumplan con lo que de buena gana propusieron. ${ }^{12}$ Porque si uno lo hace de buena voluntad, lo que da es bien recibido según lo que tiene, y no según lo que no tiene. ${ }^{13}$ No se trata de que otros encuentren alivio mientras que ustedes sufren escasez; es más bien cuestión de igualdad. ${ }^{14}$ En las circunstancias actuales la abundancia de ustedes suplirá lo que ellos necesitan, para que a su vez la abundancia de ellos supla lo que ustedes necesitan. Así habrá igualdad, ${ }^{15}$ como está escrito: "Ni al que recogió mucho le sobraba, ni al que recogió poco le faltaba". De esta manera habrá igualdad como dice la Biblia: "Al que juntó mucho no le sobró nada. Al que juntó poco, no le faltó nada".

Uno se pregunta, ¿cómo pudieron los macedonios desarrollar tal espíritu de solidaridad y generosidad que alentaba la igualdad entre ellos, y que "en medio de las pruebas más difíciles, su desbordante alegría y su extrema pobreza abundaron en rica generosidad"? Uno de los principios que podemos extraer de la experiencia macedónica es el principio de la solidaridad en la escasez. Este incidente nos muestra que la riqueza o el crecimiento de una economía local pueden resultar sostenibles, y proveer para las necesidades de todos. Las fuerzas que alientan este tipo de economía tienen un alto componente ético y de responsabilidad social. No se trata de fomentar el socialismo o el comunismo y menoscabar el capitalismo, sino de llevar a la práctica sencillos principios de redistribución de la riqueza y el crecimiento. Pablo recurre dos veces a otro principio central y fundamental como resultado de la aplicación del primero, un principio que podríamos llamar "el principio económico bíblico de la igualdad". En las circunstancias actuales la abundancia de ustedes suplirá lo que ellos necesitan, para que a su vez la abundancia de ellos supla lo que ustedes necesitan. Así habrá igualdad, como está escrito: "Ni al que recogió mucho le sobraba, ni al que recogió poco le faltaba".

\section{A manera de conclusión}

El modelo económico que pone el acento en el desarrollo básicamente individual, donde la codicia y la irresponsabilidad social juegan un rol protagónico, en pos de ganancias principalmente medidas en términos de capital monetario, ha fracasado. Como lo señala Joseph Stiglitz-Premio Nobel de Economía. 
"En esta crisis, vemos que la mayoría de las instituciones orientadas hacia el mercado en la economía, más orientada al mercado, están cayendo y acudiendo al gobierno en busca de ayuda. Todos en el mundo dirán ahora que éste es el fin del fundamentalismo del mercado. En este sentido, la caída de Wall Street es para el fundamentalismo de mercado lo que la caída del muro de Berlín fue para el comunismo" (Krugman, Stiglitz \& Soros, 2009).

La codicia, la irresponsabilidad social y la desigualdad económica, forman parte de un modelo económico basado en el lucro y el consumismo que está llegando a su fin, como lo expresó Mikhail Gorbachov, expresidente Soviético: "Ahora, esa pirámide, perniciosa e inmoral, ha colapsado. Es necesario pensar en un modelo que pueda reemplazar al actual. No estoy proponiendo romper todo sin pensar en las consecuencias. $Y$ tampoco tengo soluciones prefabricadas. El cambio tiene que ser evolutivo. Un nuevo modelo surgirá, y no puede basarse totalmente en el lucro y en el consumismo" (Krugman et. al., 2011). La crisis tal vez sea, como apuntó el presidente francés, Nicolás Sarkozy, la verdadera entrada al siglo XXI; si es así, ésta puede servir para plantear cambios en los hábitos de consumo, generar relaciones interpersonales más íntegras y llevar a cabo un desarrollo material más sostenible. Prácticas simples y cotidianas, criterios más elevados de sostenibilidad social y ambiental o la reconstitución de los lazos comunitarios pueden ser cambios positivos tras la crisis (Citado por Larrabure, 2010).

Oxfam (2011) señala que la desigualdad no solo es mala para la justicia social sino también lo es para la eficiencia económica. Stuart (2011) plantea que el crecimiento económico en los países en desarrollo es deseable y necesario, pero es la distribución de ese crecimiento lo que importa para la reducción de la pobreza. A menudo se ha promovido la expansión económica como la panacea para la reducción de la pobreza en el desarrollo de los países emergentes, sin embargo, ha habido una falta de reconocimiento de que el crecimiento es un medio para un fin, no un fin en sí mismo.

La economía solidaria no es un tema fácil de tratar y menos de ponerlo en práctica, ya que supone confrontarnos con la avaricia de los que más beneficios obtienen para que otras personas tengan una mínima oportunidad de poder progresar económicamente y favorecer su inclusión social. Una economía solidaria es posible en un mundo solidario, donde se favorece la cooperación y se promuevan iniciativas solidarias, el incidente macedónico es un caso ejemplar de una economía local orientado por principios no convencionales como la generosidad, la solidaridad y la igualdad, donde el ser 
humano es el elemento central. Para hacer esto, según Sen, el premio Nobel de Economía, hay que cambiar el enfoque y la tecnología de la economía que se utiliza para ser más sensible a los temas que tienen que ver con el bienestar y la libertad de la humanidad. Y esto requiere un verdadero compromiso (2011). Pablo menciona que los macedonios se entregaron primero a Dios e hicieron lo que Dios esperaba de ellos. La economía de la solidaridad es una respuesta a lo que Dios espera de nosotros para enfrentar el problema de la pobreza y alejarnos de la codicia y la irresponsabilidad.

Pedro Gonzales

Universidad de Montemorelos e-mail: pedrog@um.edu.mx

Recibido: 26 de agosto de 2011 Aceptado: 22 de septiembre de 2011 


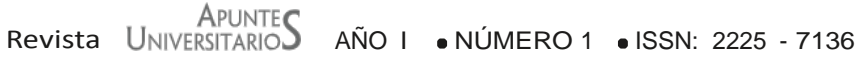

\section{Referencias}

BBC Mundo. (2009, Noviembre 24). Dos premios Nobel temen la llegada de una nueva crisis económica. Recuperado el 24 de agosto de 2011, del sitio web de la BBC Mundo, London, England: http://www.BBCMUNDO.com

De todo negocio. (2010, Agosto 18). ¿Viene una nueva crisis económica? De todo negocio. Recuperado de http://www.detodonegocios.com/inversiones/

Economía Solidaria. (2010). La economía solidaria, un nuevo concepto de la economía internacional. Recuperado el 25 de agosto de 2011, del sitio web de Economía Solidaria, Santo Domingo, República Dominicana: http://www.economiasolidaria.org/noticias/ la_economia_solidaria_un_nuevo_concepto_de_la_economia_internacional

Fondo Monetario Internacional y Organización Internacional del Trabajo (2010). Desafíos del crecimiento, el empleo y la cohesión social. Recuperado el 16 de junio de 2011, del sitio web del FMI en New York, EEUU: http://www.imf.org/external/spanish/np/sec/ pr/2010/pr10324s.htm

Goodman, P. (2008). Taking Hard New Look at a Greenspan Legacy. Recuperado el 8 de octubre de 2008, del sitio web del New York Times, New York, EE.UU: http://www.nytimes. com/2008/10/09/business/economy/09greenspan.html?_r=1\&ref=the_reckoning

Gurría, J. A. (2011, Mayo 24). ¿Un nuevo déjà vu? El economista. Recuperado de http://eleconomista.com.mx/columnas/columna-invitada-valores/2011/05/24/nuevo-deja-vu

Instituto Mundial para Investigación del desarrollo económico. (2007). Un estudio del 2\% de millonarios que posee la mitad de la riqueza mundial. Boletín de la UNU-WIDER, 44. Recuperado el 25 de agosto de 2011, de http://archive.unu.edu/update/esp/archive/issue44_22. htm

Krugma, P., Stiglitz, J., Gorbachov, M., Soros, G., M., Tofler, A., Brown, G., Oppenheimer A., y otros (2011). La crisis económica mundial. Bogotá: Publisher Oveja Negra.

Larrabure, I. (2010). El auge de la codicia. Recuperado el 25 de agosto de 2011, del sitio web de Isabel Larrabure, Barcelona, España: http://www.isabel-larraburu.com/articulos/ psicologia-social/199-el-auge-de-la-codicia.html?lang

Marcelo, J. (2009). ¿Viene una nueva crisis económica? Recuperado el 25 de agosto de 2011, del sitio web de la BBC Mundo, London, England: http://www.bbc.co.uk/mundo/economia/2009/11/091124_nueva_crisis_mj.shtml

Marcet, J. (2010). Llegó la crisis. El ABC del crack financiero mundial. México: Editorial Norma. 


\section{Pedro Gonzales}

Moon, A. (2011). Wall Street se desploma la peor ola de ventas en dos años. Recuperado el 12 de agosto de 2011, del sitio web de Yahoo Finanzas, México: http://mx.finance.yahoo. com/noticias/Wall-Street-se-desploma-peor-rlt-77199615.html?x=0\&.v=6

Moreno, M. (2011). Secretario del tesoro advierte que Estados Unidos está al borde de la quiebra. Recuperado el 7 de enero de 2011, del sitio web de elblogsalmon, Madrid, España: http://www.elblogsalmon.com/entorno/secretario-del-tesoro-advierte-que-estados-unidos-esta-al-borde-de-la-quiebra

ONU Organización de las Naciones Unidas, (2008, setiembre 23). Discurso pronunciado con motivo de la apertura del debate general de la Asamblea General de las Naciones Unidas. Recuperado el 16 de agosto de 2011, del sitio web de la ONU Nueva York, EEUU. http://www.un.org/es/ga/econcrisissummit/

Reuters. (2011). Italia, bajo el ataque de los mercados, busca apoyo político. Recuperado el 3 de agosto de 2011, del sitio web de Yahoo Noticias, España: http://es.noticias.yahoo. com/italia-bajo-el-ataque-los-mercados-busca-apoyo-143425095.html

Seisdedos, I. A. (2008). Discurso de Michelle en la ONU. Recuperado el 25 de setiembre de 2008, del sitio web de Atina Chile, Santiago, Chile: http://www.atinachile.cl/content/ view/284695/Discurso-de-Michelle-en-la-ONU.htmll

Sen, A. (2011). El desarrollo es más que un número. Recuperado el 25 de agosto de 2011, del sitio web de la BBC Mundo, London, England: http://www.bbc.co.uk/mundo/noticias/2010/11/101103_desarollo_libertad_entrevista_sen_aw.shtml

Tuck, M. (2011). Pobreza. Recuperado el 25 de agosto de 2011, del sitio web del World Bank, New York, EEUU: http://web.worldbank.org/WBSITE/EXTERNAL/BANCOMUNDIAL/NEWS SPANISH/0,,contentMDK:20550455 menuPK:1074643 pagePK:64257043 piPK:437 376 theSitePK:1074568,00.html

Word Reference. (2005). Diccionario Español. Madrid, España: Espasa Calpe. 